\title{
Communication
}

\section{Development of a Program to Fit Data to a New Logistic Model for Microbial Growth}

\author{
HIROSHI FUJIKAWA ${ }^{1 *}$ AND YOSHIHIRO KANO ${ }^{2}$ \\ ${ }^{1}$ Tokyo University of Agriculture and Technology, Faculty of Agriculture, Department \\ of Veterinary Medicine 3-5-8 Saiwai-cho, Fuchu, Tokyo 183-8509, Japan \\ ${ }^{2}$ Toyoseimaiki Corporation 12 Kuroda, Wakayama-shi, Wakayama 640-8341, Japan
}

Received 4 November, 2008/Accepted 8 February, 2009

\begin{abstract}
Recently we developed a mathematical model for microbial growth in food. The model successfully predicted microbial growth at various patterns of temperature. In this study, we developed a program to fit data to the model with a spread sheet program, Microsoft Excel ${ }^{\circledR}$. Users can instantly get curves fitted to the model by inputting growth data and choosing the slope portion of a curve. The program also could estimate growth parameters including the rate constant of growth and the lag period. This program would be a useful tool for analyzing growth data and further predicting microbial growth.
\end{abstract}

Key words : Fitting program/Logistic model/Predictive model/Microbial growth.

Recently, consumers have greatly concerned about food safety. It is of utmost importance to assure the microbiological safety of food from the production to the distribution processes. When foods are exposed to abuse (high temperatures) during these processes, contaminants grow. Once the contaminants grow in a food, they would not decrease in number even when the food is then stored in a refrigerator. If the contaminants include pathogens like Salmonella, Staphylococcus aureus, or Verotoxin-producing Escherichia coli, a food poisoning outbreak could occur. Also even if there were no pathogens, food spoilage could occur with the increased number of the contaminants. Thus, the kinetics of microbial growth in food should be analyzed for food safety.

Several growth models have been developed to describe microbial growth (Buchanan et al., 1997). The modified Gompertz model and Baranyi model are known worldwide (Gibson et al., 1987; Baranyi and Roberts, 1995). However, the former cannot predict growth at varying temperatures. The latter shows good performance, but there is an essential problem with its concept of growth in the model. That is, the

*Corresponding author. Tel : +81-42-367-5937, Fax : +81 $-42-367-5937$. model is built on the assumption that the concentration of a substance(s) critical to microbial growth (such as RNA molecule or ATP) would increase exponentially in a cell during the whole growth period (Baranyi and Roberts, 1995). However, no intracellular substances could increase exponentially during the growth period. Thus, we developed a new mathematical model that describes microbial growth based on a logistic model (Fujikawa et al., 2003). It has successfully described and further predicted microbial growth at various patterns of temperature (Fujikawa et al., 2004, Fujikawa and Morozumi 2005, 2006). It also could predict the amount of enterotoxin A produced by $S$. aureus in milk (Fujikawa and Morozumi, 2006). Predictive programs for microbial growth built on our model have been published for the food industry in Japan (Fujikawa et al., 2006b).

The new model, which originates from a logistic model, is described as follows (Fujikawa and Morozumi, 2005).

$$
\frac{d N}{d t}=r N\left\{1-\left(\frac{N}{N_{\max }}\right)^{m}\right\}\left\{1-\left(\frac{N_{\min }}{N}\right)^{n}\right\}
$$

Here $N(\mathrm{CFU} / \mathrm{g}$ or $\mathrm{CFU} / \mathrm{ml})$ is the microbial cell concentration at time $t(\mathrm{~h}), r(1 / \mathrm{h})$ is the rate constant of growth, $N_{\max }(\mathrm{CFU} / \mathrm{g}$ or $\mathrm{CFU} / \mathrm{ml})$ is the 
maximum concentration, and $N_{\min }$ (CFU/g or $\mathrm{CFU} / \mathrm{ml}$ ) is the initial concentration. $m$ and $\mathrm{n}$ are parameters related to the deceleration and acceleration phases of growth, respectively. Since our model is expressed with a differential equation, it is not easy to analyze microbial growth data with the model. Thus, we thought that a program to fit actual data to the model would be very helpful. Therefore, in this study, we developed a program to fit to our model on a spread sheet program, Microsoft Excel ${ }^{\circledR}$ 2003, the most commonly used calculation program worldwide. The fitting was done to minimize the mean of squared errors, MSE, between the measured and estimated cell concentration (in log units) during the observation period. Calculation was performed to estimate parameters $m$ and $n$ that give the least MSE with Exce ${ }^{\circledR}$ Solver. The program has been also developed so that it does not require any special knowledge

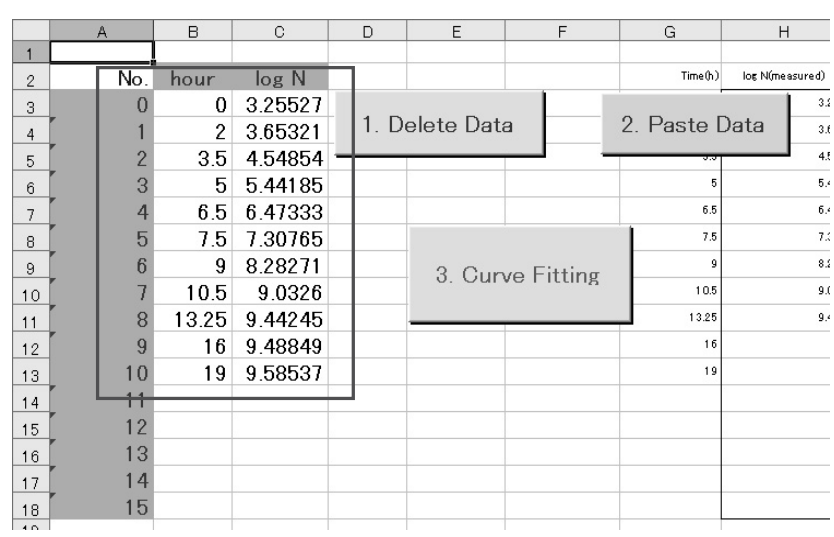

FIG 1. Data input to the fitting program. A square region shows a growth data set consisting of time and the cell numbers (in log unit).

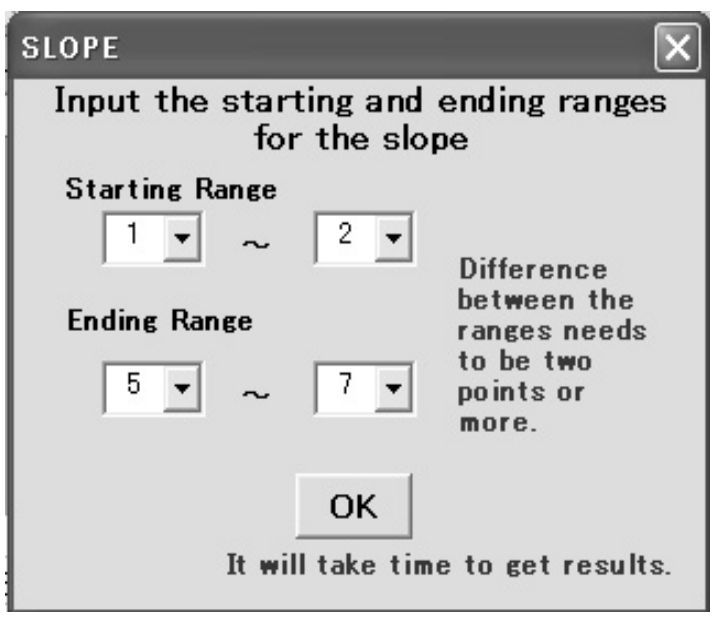

FIG 2. Input window for the slope. about the mathematical model and computer program.

The procedures for the data fitting program are as follows. First, we need to input raw growth data that consists of a set of storage hours and cell numbers (in log units). To do this, the fitting program has two buttons (Fig. 1). By pushing the Delete Data button, all data that we have previously input are removed. After copying a new data set, we can paste it with the Paste Data button; see the data set in the square in FIG. 1. Here the maximum number of the data set for analysis is thirty-two. FIG. 1 shows growth data of $E$. coli that grew in mashed potato samples at $30^{\circ} \mathrm{C}$ (Fujikawa et al., 2006a).

Second, push the Curve Fitting button to fit and analyze the data. Then a window that requires inputting the slope position in a growth curve will appear on the screen (FIG. 2). A growth curve is commonly a sigmoidal one with a slope, or the logarithmic phase. For the curve fitting, the position of the slope is essentially important. Thus, we input the candidates for the starting point and ending point of the slope on the screen. In FIG. 2, we selected serial data points from the first to the second point in the data set of FIG. 1 for the starting point of the slope. Also, we selected serial points from the fifth to the seventh data points for the end of the slope. The distance between the starting point and the ending one needs to be marked by two points or more.

On pushing the OK button, the program starts fitting the data. With each combination of the starting and ending points, a curve fitted to the growth data is generated. Results for all combinations are shown in a separate sheet (FIG. 3). The value of MSE between measured and estimated cell concentration (in log units) during the observation period is given for each fitted curve. Among the curves, the curve with the smallest MSE is chosen as the best-fitted curve. The smallest MSE value is highlighted on the screen (FIG. 3); a curve with the slope from the first point to the sixth point is the best-fitting curve in the figure. The best-fit curve then appears on the screen of the fitting program (FIG. 4). The characteristics of the curve such as the correlation coefficient of the slope, the rate constant of growth $(r)$, the duration of the lag period, and the value of MSE for the observation period are also shown (FIG. 4).

The program could show good fitting performance, as seen in FIG. 4. Some of other fitting results are also shown in FIG. 5. Thus, program users easily can get a growth curve fitted to their raw data. Furthermore, they can compare the curve with others originating from different data. These analyses would be useful to quantitatively study microbial growth in 


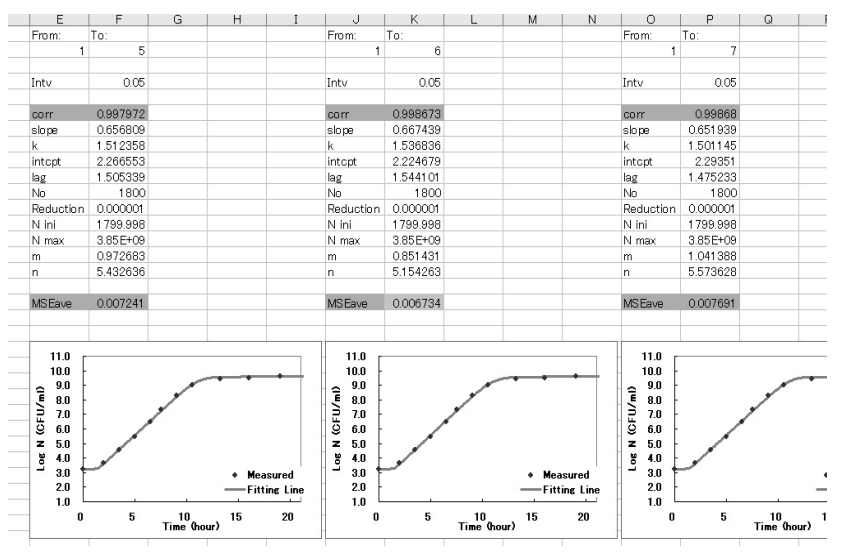

FIG 3. Candidates for the best-fit curve. Results of all combinations for the slope are presented in the sheet.

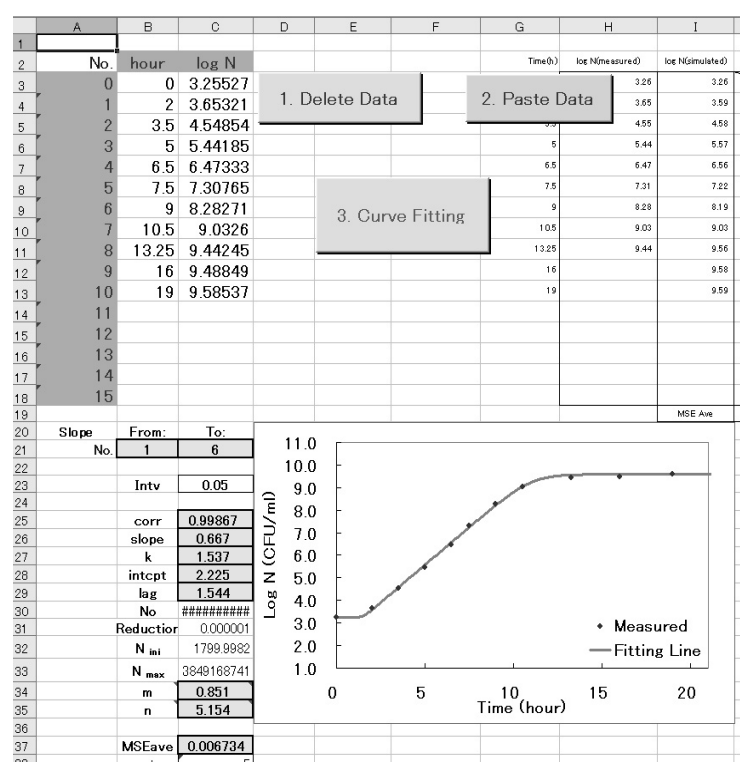

FIG 4. Results of the fitting analysis. The best-fit curve and its characteristics are shown on the screen.

food products and then lead to the risk analysis for microbial food poisoning outbreaks and food spoilage.

After users analyze their data at various constant temperatures with the fitting program, they can further predict microbial growth at various temperature patterns. That is, with the values of $k$ at the constant temperatures, they can make the Arrhenius plot, which can estimate the value of $k$ at a given temperature. With the estimated value of $k$, our logistic model can predict microbial growth at various temperature patterns (Fujikawa et al., 2003 and 2004; Fujikawa and Morozumi 2005, 2006).

We confirmed that our fitting program works well in this study, but the most important thing to analyze
A.

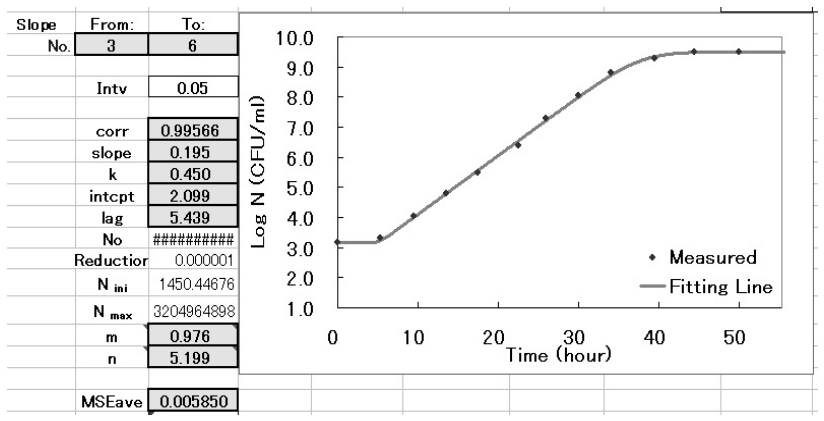

B.

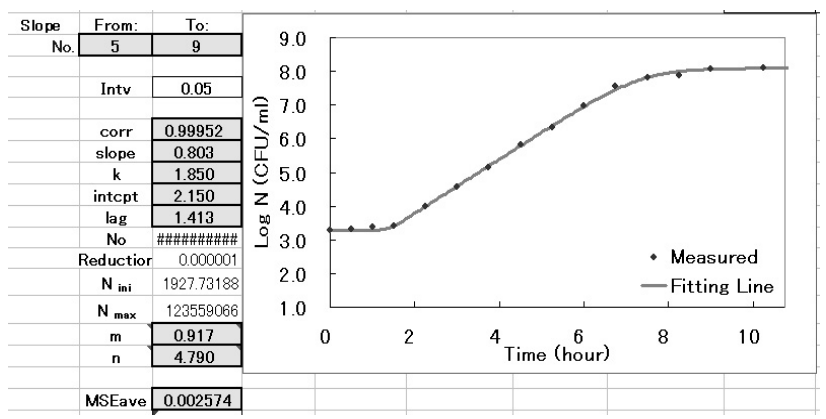

FIG 5. Examples of curves fitted with the program. Raw growth data obtained by the author were analyzed. A: $E$. coli growth in a pouched mashed potato at $18^{\circ} \mathrm{C}$ (Fujikawa et al. 2006a). B: S. aureus growth in milk at $36.5{ }^{\circ} \mathrm{C}$ (Fujikawa and Morozumi 2006).

and predict microbial growth is whether we can obtain good raw data or not. Our program merely fits the data. If the data is not accurate, the fitted curve is not reliable when used for the analysis of food safety. Thus, we need to get as accurate raw growth data as possible.

\section{ACKNOWLEDGMENT}

The authors thank Ms. Yuki Watanabe for her technical assistance in computer programming.

\section{REFERENCES}

Baranyi, J., and Roberts, T. A. (1995) Mathematics of predictive food microbiology. Int. J. Food Microbiol., 26, 199218.

Buchanan, R. L., Whiting, R. C., and Damert, W.C. (1997) When is simple good enough: a comparison of the Gompertz, Baranyi, and three-phase linear models for fitting bacterial growth curves. Food Microbiol., 14, 313326.

Fujikawa, H., Kai, A., and Morozumi, S. (2003) A new logistic model for bacterial growth. J. Food Hyg. Soc. Japan, 
44, 155-160.

Fujikawa, H., Kai, A., and Morozumi, S. (2004) A new logistic model for Escherichia coli at constant and dynamic temperatures. Food Microbiol., 21, 501-509.

Fujikawa, H., and Morozumi, S. (2005) Modeling Surface Growth of Escherichia coli on Agar Plates. Appl. Environ. Microbiol., 71, 7920-7926.

Fujikawa, H., and Morozumi, S. (2006) Modeling Staphylococcus aureus Growth and Enterotoxin Production in Milk. Food Microbiol., 23, 260-267.

Fujikawa, H., Yano, K., and Morozumi, S. (2006a)
Characteristics and Modeling of Escherichia coli Growth in Pouched Food. J. Food Hyg. Soc. Japan, 47, 95-98.

Fujikawa, H., Yano, K., Morozumi, S., Kinura, B., and Fujii, T. (2006b) Development of a predictive program for microbial growth under various temperature conditions (in Japanese) J. Food Hyg. Soc. Japan, 47, 288-292.

Gibson, A. M., Bratchell, N., and Roberts, T. A. (1987) The effect of sodium chloride and temperature on the rate and extent of growth of Clostridium botulinum type $A$ in pasteurized pork slurry. J. Appl. Bacteriol., 62, 479-490. 